\title{
Kinect-Based Real-Time Acquisition Algorithm of Crop Growth Depth Images
}

\author{
Shan Hua (D, Minjie Xu, Zhifu Xu, Hongbao Ye, and Cheng quan Zhou \\ Key Laboratory of Creative Agriculture, Ministry of Agriculture and Rural Affairs, Institute of Agricultural Equipment, \\ Zhejiang Academy of Agricultural Sciences, Hangzhou 310021, Zhejiang, China \\ Correspondence should be addressed to Shan Hua; huashan@zaas.ac.cn
}

Received 20 May 2021; Revised 9 June 2021; Accepted 2 July 2021; Published 21 July 2021

Academic Editor: Sang-Bing Tsai

Copyright (c) 2021 Shan Hua et al. This is an open access article distributed under the Creative Commons Attribution License, which permits unrestricted use, distribution, and reproduction in any medium, provided the original work is properly cited.

Kinect 3D sensing real-time acquisition algorithm that can meet the requirements of fast, accurate, and real-time acquisition of image information of crop growth laws has become the trend and necessary means of digital agricultural production management. Based on this, this paper uses Kinect real-time image generation technology to try to monitor and study the depth map of crop growth law in real time, use Kinect to obtain the algorithm of crop growth depth map, and conduct investigation and research. Real-time image acquisition research on crop growth trends provides a basis for in-depth understanding of the application of Kinect real-time image generation technology in research. Kinect image real-time acquisition algorithm is a very important information carrier in agricultural information engineering. The research results show that the real-time Kinect depth image acquisition algorithm can obtain good 3D image data information and can provide valuable data basis for the 3D reconstruction of the later crop growth model, growth status analysis, and real-time monitoring of crop diseases. The data shows that, using Kinect, the real-time feedback speed of crop growth observation can be increased by $45 \%$, the imaging accuracy is improved by $37 \%$, and the related operation steps are simplified by $30 \%$. The survey results show that the crop yield can be increased by about $12 \%$.

\section{Introduction}

With the continuous and rapid development of global agricultural informatization, digital modern agriculture has gradually developed into premature and become a new development trend of modern agriculture in China [1]. The digital modern agricultural system is an agricultural hightech system that integrates modern agricultural engineering science, environmental science, computer information technology, communication information technology and international network information technology, and many other modern scientific information technologies into one. It is also a new agriculture in which modern agriculture in China is flourishing [2]. As a main key technology research and development direction in the agricultural and food field of the current digital era, the monitoring technology of crop plant growth morphological changes has played an important leading role in the entire development process of agricultural food production in China [3]. Before the advent of Kinect technology, the remote sensing space monitoring of crop areas has always been regarded as an important technical topic in the application of remote sensing space in China, but the current remote sensing space monitoring technology mainly uses remote sensing satellites for space remote sensing monitoring. In fact, it is subject to changes in local climate and meteorological conditions [4].

The traditional main methods for monitoring the growth potential and morphological maps of other crops are obtained through cumbersome manual automatic measurement or using expensive laser image data collection and monitoring equipment, such as using CCD laser cameras, digital cameras, light wave image scanners, and binocular monitoring equipment [5]. Therefore, in order to effectively solve some of the shortcomings of the above two traditional somatosensory monitoring experimental methods, the research project in this paper uses a self-developed indoor and outdoor somatosensory real-time game, human activity parts, and plant posture movements developed by Microsoft 
Recognition and other aspects of the cheap indoor somatosensory real-time game monitoring equipment Kinect and tried an extended and extended real-time somatosensory monitoring that can be applied to the growth pattern of outdoor indoor crops [6]. It can also be used as a panoramic camera combining somatosensory machines and game consoles as recording hardware. Kinect not only supports real-time automatic detection and real-time picture transmission, but also can automatically identify and complete a series of complex actions [7]. The base motor can drive the whole Kinect to rotate in the left and right direction at the same time, so that it also has the important function of highspeed dynamic image capture. Therefore, Kinect can possess the powerful transmission function of capturing color image, $3 \mathrm{D}$ light and depth image information at the same time, and sonic video signal [8].

We explore the role of Kinect depth image real-time acquisition algorithm in crop growth observation. Among them, $\mathrm{Wu}$ et al. made a detailed introduction to Kinect image imaging technology, analyzed the current problems in the development of Kinect image imaging technology, and elaborated related research methods [9]. In their article, Jia et al. proposed the research significance and research status of real-time capture of Kinect depth images, explained the basic theory of Kinect for real-time capture of depth images, and showed the significance and importance of data processing and monitoring of real-time capture of Kinect depth images [10]. Huang et al. elaborated on the way and method of real-time image acquisition algorithm of Kinect depth image transmission in crop growth observation and proposed the advantages and disadvantages of the Kinect algorithm for acquiring crop depth image [11]. Raviya et al. proposed the efficiency and accuracy of traditional crop growth monitoring methods, pointed out the feasibility of real-time Kinect depth image acquisition algorithm for crop growth detection, and proposed a variety of three-dimensional image data processing algorithms based on the Kinect algorithm, in addition to the advantage of real-time acquisition algorithm of depth image [12]. However, the research did not use artificial intelligence methods, so the research content is insufficient.

The main research content of this article is roughly divided into five parts. The first part is the introduction, aiming to systematically review the main research topics of this article from the research background, research purpose, research ideas, and methods $[13,14]$. The second presents Kinect depth image real-time acquisition technology current research principles, details, and system summary. The third part is related technology, introducing the technology and principle used by Kinect in the system development process, including the principles and technologies related to Kinect, as well as the principles of 3D model driving. The fourth part is the analysis of the technical requirements for real-time acquisition of Kinect depth images. The requirements of the Kinect image acquisition algorithm are described from two aspects: functional and nonfunctional. The fifth part is the construction and implementation of Kinect system. It is an overview of Kinect network construction, Kinect network modification, Kinect data filtering application program, and further discussion and application prospects of real-time acquisition algorithm of Kinect depth image. It has obtained good three-dimensional image data information and can provide valuable information for the three-dimensional reconstruction of crop growth models, analysis of growth status, and real-time monitoring of diseases and insect pests (data basis).

\section{Theoretical Basis}

2.1. Kinect Basic Description and Related Algorithms. Kinect 3D imaging technology, like many other emerging imaging technologies, was originally believed to be directly derived from the national military application field [15]. With the rapid development of national information and industrial modernization, the important application and potential of Kinect 3D imaging technology have improved in various fields gradually. It appears that Kinect 3D imaging technology itself has experienced the development process from the beginning of birth to the initial rapid development period to the stable and gradual development period to the economic prosperity period. Using computer graphics to conduct virtual natural scientific research on crop wheat leaves and to simulate the natural growth morphology of a crop wheat leaf in three-dimensional space, a natural mathematical form that can simulate a crop wheat leaf through twisting and wheat curling is proposed by the model, which can flexibly control the effect of wheat leaves after twisting and wheat curling, showing the natural growth morphology of crop wheat leaves under natural conditions $[16,17]$. After the image is generated by Kinect, a two-dimensional image simulation area is automatically created, including two gradient matrices; the horizontal matrix is a, the vertical matrix is $b$, the matrix size is represented by $\mathrm{S}$, and the sober operator is an improved first-order numerator. Using this calculation, the child can calculate the image edge gradient $R$, so a set of varied Kinect picture combinations will be obtained. For the Kinect depth image real-time acquisition algorithm's image generation formula, where $E$ is the picture function filter and $(u v)$ is the matrix threshold, the operation is as formula (1)-(3) shown as follows:

$$
\begin{aligned}
R(a, b) & =1-\sqrt{\frac{\sum[S(a)-S(b)]^{2}}{a-b}}, \\
e^{x} & =\sum_{k=0}^{n} \frac{x^{k}}{k !}+R_{n}(X), \\
(u v) & =\sum_{k=0}^{n} C_{n}^{k} u^{n-k} v^{(k)} .
\end{aligned}
$$

The Kinect depth camera can simultaneously output the distance from the camera to the pixels while capturing video. This kind of picture is called a depth image. The Kinect depth image is to draw the graphics or images obtained by the three-dimensional sensor to obtain the three-dimensional shape of the object and perform image analysis and processing-what the scanning device does not have. The 
research based on Kinect depth camera can be divided into 5 categories: object recognition, 3D object reconstruction, robot navigation, augmented reality, image processing, and human-computer interaction. Although traditional three-dimensional laser scanners and structured light scanners can accurately obtain three-dimensional information on the surface of objects, such devices are not easy to operate, have complex structures, and are expensive. Although a stereovision system composed of multiple color cameras can obtain three-dimensional spatial information at a low cost, such system algorithms have defects to a large extent, such as low efficiency, high computational complexity, and difficulty in processing. The case of texture: the Kinect plant microscopic change detection processor will automatically generate an operation interval, which is to complete the conversion of the same name point of the $2 \mathrm{D}$ image to the same name point of the $3 \mathrm{D}$ point cloud data by extracting the feature of the image coordinate factor and to determine the pose of the adjacent frame of Kinect, where the three-dimensional space coordinates include $(x, y)$, and the simulated Kinect processor operation formula is as shown in formulas (4)-(6), where $d$ augments the operation factor of the function and $x$ is the base of the Jacobian matrix:

$$
\begin{aligned}
f(x, y) & =\lim _{x \longrightarrow 0} \frac{f(x, y)}{x^{2}+y^{2}}, \\
\int \frac{\mathrm{d} x}{a x+b} & =\frac{1}{a} \ln |a x+b|+c, \\
I_{x} & =\iint_{\Omega}\left(y^{2}+x^{2}\right) p \mathrm{~d} v .
\end{aligned}
$$

Kinect's real-time image processing uses a computer algorithm to establish a crop growth point recognition model and extracts and calculates the differences in crop growth. Before building the model, Kinect performs different operations in space and samples at different degrees of occlusion and then manually marks crop nodes to form a sample analysis library. Then, use a machine learning algorithm to train the sample analysis library. Kinect's core technology is bone tracking, and its hardware foundation is a depth camera. However, the depth imaging principles in the first- and second-generation Kinect are different. The first generation uses optical coding ranging technology, and the second generation uses time-of-flight ranging technology. This illuminates the plant surface and penetrates the frosted glass plate, forming a random diffraction. The first-generation Kinect uses an optical coding distance technology that is different from traditional optical coding distance technology because it directly controls a continuous infrared light source instead of a pulsed light source $[18,19]$. The spots are called laser spots. This kind of spot has a high degree of randomness, and because the spots formed in different spatial positions are different, the entire space is equivalent to a mark, and the spots can be analyzed and decoded to identify any points you can get (position in $\mathrm{H}$ dimensional space). Therefore, Kinect estimates the distance of each point in space in the depth camera. The depth camera principle of Kinect's time-of-flight ranging technology is simple, but the hardware accuracy requirements for its implementation are relatively high.

\subsection{Basic Principle of Real-Time Acquisition Algorithm of} Kinect Depth Image. Kinect image is a kind of intuitive information. Kinect image real-time acquisition algorithm is a very important information carrier in agricultural information engineering. However, current mainstream agricultural research image objects generally have the following formats: visible light images, infrared images, remote sensing images, multispectral/hyperspectral images, radar images, etc. With the emergence of Kinect, you can easily obtain real-time depth images. Since the image generated by the Kinect algorithm contains 3D information, Kinect expands the path of agricultural resources and allows users to obtain more information for applications. Traditional crop observation methods cannot meet the needs of agricultural production.

Kinect can also identify subtle changes in crop growth. If the monitored crop grows abnormally, it will alarm in real time. However, this kind of behavior detection technology can only be easily applied in the Kinect monitoring system. It will be expanded in the future. More examples can be added to the crop identification library and can have a certain growth prediction function. The function that the crop growth module needs to achieve is to identify the crops within the detection range of Kinect and determine whether it violates the growth trend. In order to ensure the effectiveness of the identification of crop growth in the monitoring system, this paper uses a multichannel tracking identification method. In the existing tracking-recognition algorithm, improvements are made. Kinect is a relatively good tracking algorithm. Continuous learning is to obtain the latest appearance features of the target, so as to improve tracking in time and achieve a better tracking effect. Compared with other tracking algorithms, the biggest advantage of Kinect tracking algorithm is that it can track the target. But even the TLD algorithm has shortcomings. In the TLD algorithm, the knowledge used mainly comes from the sequence of video frames with timing relationships, which limits its learning of spatial information to a certain extent. Kinect's depth camera can just improve its shortcomings. By transferring the depth data captured by Kinect to the learning module and tracker, it can improve the accuracy and usability of the TLD algorithm to a certain extent [20]. Gray scale linear transformation is a commonly used processing method for enhancing images. It is actually in the pixel space, using point arithmetic to improve the gray-scale contrast of the image. Linear transformation is usually divided into overall gray linear transformation and local gray linear transformation. The mathematical expression of gray linear transformation is shown in formulas (7)-(10), where the gray value of Kinect picture is the stretching curvature of the picture. $\mathrm{FN}$ is the median filter. 


$$
\begin{aligned}
\int x^{2} \mathrm{~d} x & =\frac{x^{a+1}}{a+1}+C \\
d & =\frac{\left|A x_{0}+B y_{0}+C_{0}+D\right|}{\sqrt{A^{2}+B^{2}+C^{2}}}, \\
\mathrm{FN} & =L * \operatorname{snb}\left[\sum_{i=1}^{n l}\left(y_{i}^{n}-f y_{i} y_{l}+y_{l}^{n}\right)+\sum_{i m+1}^{n_{l}+n_{R}}\left(y_{i}^{n}-f y_{i} y_{R}+y_{R}^{n}\right)\right], \\
V_{E} & =V_{A} N\left(d_{1}\right)-e^{-r t} D N\left(d_{2}\right) .
\end{aligned}
$$

More examples can be added to the crop recognition database, which can have a certain growth prediction function. The function that the crop growth module needs to realize is to identify the crops within the detection range of Kinect and judge whether it violates the growth trend.

\section{Setup of Crop Growth Test Experiment}

3.1. Kinect Image Acquisition Experimental Conditions. This article about the Kinect experiment foundation is based on the capture of crop pictures. For a known Kinect, the effective capturing angle of view in the horizontal direction is about 80 degrees, and the research shows that the credibility of the crop node data obtained when the angle between the crop and Kinect exceeds 40 degrees is less than $5,000 \%$, which is basically impossible use. In addition, in order to ensure a smooth transition between Kinect during correction and relay, there is an effective capturing area of approximately 10 degrees between Kinect pairs. Therefore, this week's experiment requires a total of 6 Kinects to achieve 360-degree motion capture. In the layout of the Kinect network hardware, we placed 6 Kinects in a regular hexagon shape, with a height of $80 \mathrm{~cm}$ from the ground. Each Kinect is also $350 \mathrm{~cm}$ away from the regular hexagon. The Kinect camera points to the middle of the regular hexagon to form a positive six with a length of $360 \mathrm{~cm}$ (effective capture area for polygons).

Because the encoding technology of high-depth laser images obtained by our Kinect and others is different from our traditional lit of firefight or unstructured light image measurement encoding technology, it directly uses a coding technology we call light image encoding (light coding). The laser used is continuous laser illumination (nonphotosensitive pulse), so our experiment only needs ordinary kismets and other photosensitive element chips to carry out very smoothly, which may greatly reduce the technical cost. In this experiment, the main components of the depth detection imaging laser system include three main core photosensitive elements: high-speed laser imaging emitter, nonuniform transparent photosensitive medium, and cods and other photosensitive imaging devices. The laser imaging emitter and the photosensitive device such as ammos together form a certain photosensitive angle to align the target imaging scene, and the uneven transparent photosensitive medium is placed in front of the lens on a laser imaging emitter. The laser spot emitter can randomly emit diffuse laser through an uneven optical medium, and laser speckle (laser speckle) can also be formed in some scenes, that is, when the diffracted laser spot irradiates a rough mesh or the laser penetrates to random diffusion diffraction laser spots formed when cilia glass is behind. These spatial speckle patterns will change with the shooting distance to produce different speckle patterns, so the camera must first calibrate the three-dimensional spatial spots before shooting; that is, take a three-dimensional reference projection plane every few seconds and take the speckle pattern on this reference projection plane which is recorded. Kinect is to project some "random" dot matrix through the infrared microwave transmitter element, and then we only use a common klismos infrared sensor element to accurately capture this infrared dot matrix. When the input depth of the output scene changes, the output dot matrix captured on the camera will naturally change. Through these dot matrix changes, the information on the depth of the output scene can be accurately measured.

3.2. Kinect Image Acquisition Experiment Content. In this paper, an experimental system that can control any position in real time according to the growth of crops is selected, and 30 groups of static scenes of medium-sized crop radishes are selected as experimental objects. The system uses Kinect to identify the omnidirectional position of crops, to identify the subtle differences between crops, and to make corresponding instructions to the intelligent system through different forms of crops. The intelligent system can move accordingly after receiving different position commands, thereby constructing different patterns. Kinect 3D depth imaging image reconstruction obtains the effect of combined algorithm detection. The detection effect depends mainly on $3 \mathrm{D}$ image reconstruction. This paper studies and constructs a system consisting of 2 Kinect monitoring systems to perform 3D image observation on the overall growth of collected crops. In this monitoring system, first of all, by using a checkerboard grid three-dimensional calibration method, two Kinect systems are calibrated three-dimensionally; secondly, by using the Kinect in two units, a threedimensional scan of the comprehensive detection data of each crop growth within a detection data range can be obtained and corresponding detection data is finally combined with the $3 \mathrm{D}$ point cloud point map data of each leaf of 
the crop and the 3D calibration detection result data collected by using the superimposed second-generation nearest point scanning algorithm, so as to obtain more complete 3D point maps and cloud data models, etc. By analyzing and using Kinect system parameters to refine the system graphic symbols, simplify the combination algorithm of system graphic symbols, and combined with the specific characteristics of crop growth, the crop image information is collected, and a three-dimensional image of crop growth is constructed to realize the three-dimensional visualization of the crop growth management process.

The content displayed is how to use Kinect to obtain a two-dimensional color of various eggplant growth potentials in different forms in outdoor real-time algorithm images and 3D depth color images. This article can be used as a laboratory object to perform an algorithm simulation test of an outdoor real-time color acquisition method for two-dimensional deep color images. The point on the left is the color image. The right is the image bit depth calculation image corresponding to the point. The image bit resolution is uniformly $640 \times 480$, and the image bit depth of field is 16 bits. The points on the color pixels should have a different depth value from 0 to 65535 , and the different depth values represent the average distance that this pixel can reach 2 Kinects. When the value of the depth difference is 0 , the display is usually white or black, indicating that the position is the closest or farthest without a significant depth difference; when the value of the display depth difference is usually $0 \sim 65535$, the display is usually black or white, indicating the distance. The depth value of Kinect is the closest or longest distance that the camera position can be supported. The test results of this method show that the 3D depth dynamic images obtained by real-time dynamic image acquisition algorithms such as kinkiness are relatively clear and the analysis effect is good, which can provide real-time reference resources for the related research work such as ecological analysis of crop planting growth and 3D ecological reconstruction.

\subsection{Kinect Image Acquisition Algorithm Experimental Data} and Experimental Significance. Through this experiment, it is known that Kinect has performed RGB image segmentation and Kinect cluster segmentation on color images. Compared with a single segmentation method, the average segmentation error is reduced by 12.12 and 41.48 percentage points, respectively, and obtained a higher accuracy. Picture clarity increased by $40 \%$. For the depth image, the corresponding color image is used as the reference matching target, and the missing and erroneous depth data are repaired by the Kinect nearest neighbor regression algorithm multiple times, and the repair success rate is as high as $97.15 \%$. For the detection of crop plant images in the dynamic process, a platform for simulating vehicle plant detection was designed. The test adopts the way of Kinect sensor adaptive vehicle traveling speed, and the dynamic test is carried out in the vehicle speed range of $0.1 \mathrm{~m} / \mathrm{s}$ to $1.0 \mathrm{~m} / \mathrm{s}$, focusing on the analysis of the collected images. For color images and depth images, the quality of the collected color images decreases with increasing vehicle speed, while depth images are basically not affected by speed. This experiment is also applicable to image plant detection methods for dynamic images and can reduce the impact of vehicle speed on color image quality and improve the clarity of Kinect transmission images. The node information table structure of the sensor used in this paper is shown in Table 1.

The experimental results show that the Kinect depth sensor can output RGB images and $320 \times 240$ infrared depth images at a resolution of $640 \times 480$ and at the same time obtain 3D spatial information of indoor scenes at a frequency of $30 \mathrm{fps}$. The optimal distance for the Kinect depth sensor to measure the environment or object is $1.2 \mathrm{~m}$ to $3.5 \mathrm{~m}$, the horizontal viewing angle is $57^{\circ}$, and the vertical range is $\pm 28^{\circ}$. The accuracy of the depth data acquired by the Kinect depth sensor is limited by the scanning distance between the reconstructed object and the sensor. In theory, if the distance between the reconstructed object and the sensor is in the range of $0-600 \mathrm{~mm}$, the accuracy of the depth data can reach $1 \mathrm{~mm}$. On the other hand, if the distance between the reconstructed object and the sensor is in the range of $600-1000 \mathrm{~mm}$, the accuracy of the depth data drops to about $3 \mathrm{~mm}$. If the distance between the reconstructed object and the sensor is less than $2000 \mathrm{~mm}$, ensure that its accuracy is less than $10 \mathrm{~mm}$. In the field of independent positioning and indoor 3D mapping, Kinect depth sensors are also being studied in detail. In this experiment, depth information and visual information are combined to realize the reconstruction of the crop 3D map. The significance of this experimental study is a rapid reconstruction method of indoor 3D spatial information based on the Kinect depth sensor. By comparing and analyzing the influence of various external factors on the modeling accuracy, a 3D data collection scheme for indoor spatial information based on the Kinect depth sensor is summarized. The corresponding accuracy analysis of the obtained point cloud model is carried out.

\section{Analysis of the Results of Crop Growth}

4.1. Comparative Analysis of Kinect-Based Real-Time Acquisition Algorithms of Crop Growth Depth Images. Compared with other traditional three-dimensional visual scanning method equipment algorithms, the Kinect stereo deep color image information acquisition scanning algorithm not only has an information structure that can accurately capture the color and dark color of the internal surface of the illuminated object in real time, but also is smaller and easier to use than the Kinect scanning equipment low price); compared with the equipment of stereoscopic three-dimensional vision scanning method, the working principle of Kinect is mainly to actively emit near and outside light, which is not easy to be affected by changes in infrared light intensity and changes in the color and texture of the internal surface of the illuminated object relatively low. In addition, binocular video recognition sometimes requires real-time matching of the data of two mobile cameras shooting the same video scene separately. 
TABLE 1: Sensor node information table.

\begin{tabular}{lcccc}
\hline Induction value & POSFS & CEFS & PSORSFS & CIQFS \\
\hline Tic-tac-toe & 25 & 7 & 9 & 101 \\
Breast-cancer & 36 & 5 & 9 & 152 \\
Vote & 44 & 10 & 6 & 218 \\
Mushroom & 62 & 3 & 12 & 269 \\
\hline
\end{tabular}

The computer capacity is relatively large and it is difficult to fully meet the real-time recognition requirements. By using the Ki Kinect method to obtain binocular depth recognition images, it is possible to effectively solve the complex problems encountered by the above two monocular and other binocular image recognition methods. The comparison of the two crop detection methods is shown in Figure 1.

The main advantages of Kinect are as follows. (1) It can quickly obtain the depth information of objects and scenes, which meets the needs of real-time monitoring. (2) The pixel gray value is only related to the distance between the object or scene and the depth camera and is not subject to light. Influenced by factors such as shadows or shadows, it can adapt to various environmental changes and is robust. (3) The core equipment is cheap color cameras, infrared emitters, and infrared CMOS sensors, and the use is similar to ordinary cameras, suitable for universal use. (4) In addition to being able to acquire depth images, Kinect devices can also obtain corresponding RGB color images, which is more conducive to the analysis and processing of later graphics and images. The comparison data of Kinect picture acquisition algorithm in the study is shown in Table 2.

Kinect real-time image acquisition algorithm for crop growth observation can convert the collected information into depth information. In order to reduce the difficulty of visual matching, two Kinect cameras that are parallel to each other or aligned up and down can be used to acquire the perception image of the same object from two different observation points. In this way, the peripolar geometry is used to transform the problem into Euclidean geometry. Assuming that the optical axes of the two cameras are strictly parallel, triangulation is used to convert the parallax information of the matching points into depth information. For Kinect real-time image acquisition algorithm in order to more accurately grasp the crop growth information, this article is divided into the following six steps for research and observation: image acquisition, camera calibration, feature extraction and matching, camera correction, stereo matching, and three-dimensional modeling. Concerning image acquisition, to simplify the calculation of camera calibration, two cameras are used to acquire images from different viewpoints. Two cameras are required to meet the requirements of left-right or left-right parallel alignment as much as possible. At the same time, necessary preprocessing is performed, such as smoothing, noise reduction, and image enhancement. Camera calibration includes calibration of camera internal and external parameters and calculation of camera perspective projection matrix. At the same time, the radial, tangential, and centrifugal deformation of the camera is eliminated. In order to obtain the camera position relationship and perform stereo correction, feature extraction

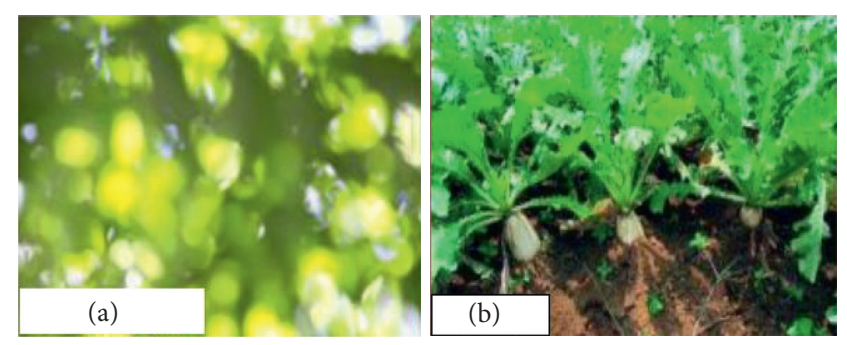

FIgURE 1: Comparison of traditional methods with Kinect images. (a) Traditional way. (b) Kinect.

and matching need to adopt an appropriate matching method according to the specific conditions of the object. These Kinect core technologies are the necessary conditions to meet crop observations, which can improve agricultural production efficiency and minimize useless work. The realtime acquisition algorithm of Kinect depth image is used for the data obtained from the detection of crop growth, as shown in Figure 2.

As can be seen from the data in Figure 2, the real-time acquisition algorithm of Kinect depth images for crop growth detection can shorten the time of abnormal detection and greatly improve the detection efficiency, and the detection efficiency is $22 \%$ higher than the general processing method.

Based on the Kinect three-dimensional skeleton, this paper proposes a sequence motion feature model about plant angle changes. The model uses the subtle situation in the process of crop rhizome changes as the entry point, and the rotation angle of the Kinect camera determines the observation position. By using the depth image information obtained by the Kinect device, Kinect is segmented from the background, and then 3 expressions are added by the moment to make it contain more detailed characteristics, so as to identify the image target, and finally the recognition result is converted. The control instructions are transmitted to the intelligent system, and finally the whole process control is realized: taking the metamorphic rhizomes of crops as objects, using the Kinect surface reconstruction method to obtain the color cloud points of their metamorphic rhizomes, performing registration, filtering, and surface reconstruction on the color point cloud data to extract the length parameters between the feature points in the surface model and metamorphic rhizome volume parameters, and conducting statistical analysis for verification. The results show that the surface reconstruction models can fully display the texture features of their metamorphic rhizomes, the length parameters, and volume configuration parameters between the extracted feature points, and the errors do not exceed $10 \%$. Kinect depth image real-time acquisition algorithm is used for crop growth and detection system sensitivity data, as shown in Figure 3.

From the data in Figure 3, the real-time acquisition algorithm of Kinect depth images is used for crop growth. Its intelligent detection feedback system will greatly improve its sensitivity, and the degree of sensitivity improvement is about $30 \%$. 
TABLe 2: Picture transmission data record form.

\begin{tabular}{lccc}
\hline Serial number & Acquisition cycle & Number of objects & Number of attributes \\
\hline 1 & Tic-tac-toe & 955 & 52 \\
2 & Lymphography & 146 & 65 \\
3 & Vote & 300 & 12 \\
\hline
\end{tabular}

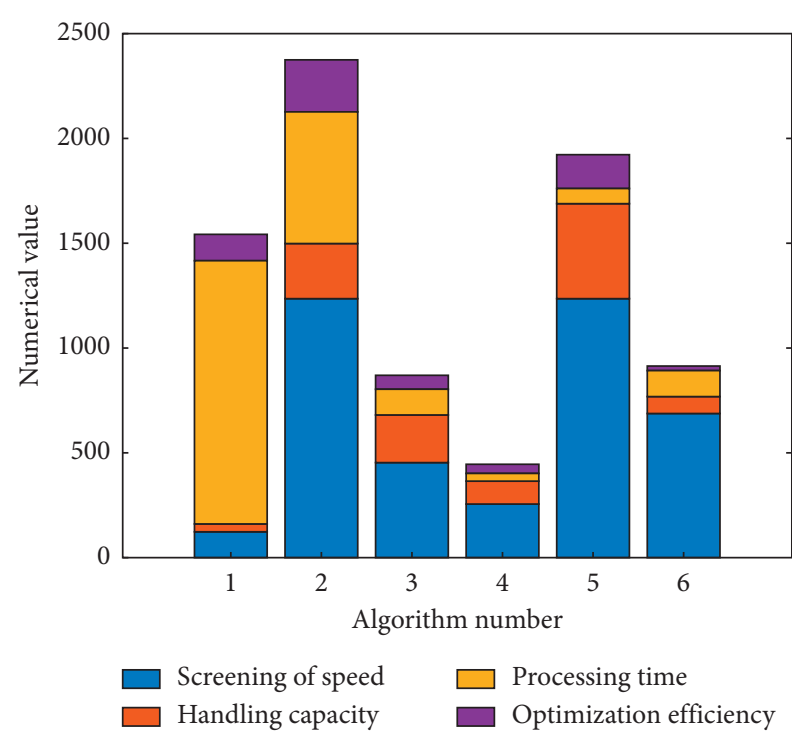

FIGURE 2: Increased crop feedback efficiency.

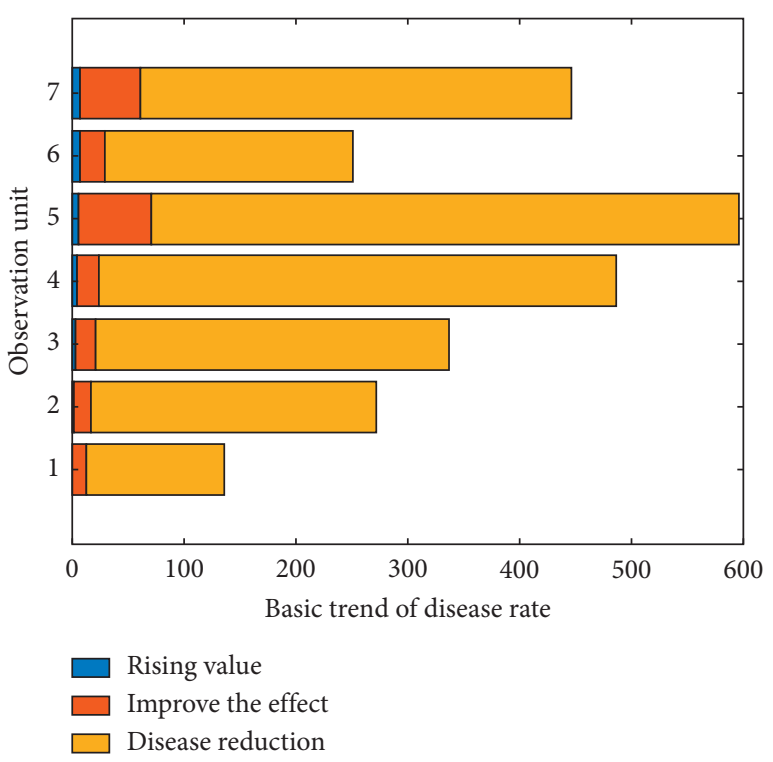

FIGURE 3: Crop growth sensitivity has been greatly improved.

4.2. Kinect-Based Application Analysis of Crop Real-Time Depth Image Acquisition Algorithm in Real Time. This article focuses on the complete application of Kinect depth image acquisition algorithm in agricultural production. In precision agricultural production, information about crop growth is the key to automated agriculture. In agriculture in automatic farming, automatic tillage machines follow Kinect to obtain agricultural operation instructions, such as the direction and distance of wheels, angle and intensity of automatic operation, and other data sent from the Kinect system for processing and analysis. We will access important information. In the agricultural production process, the shape of crops is not constant, and the color and position are not fixed or standardized. Therefore, Kinect's computer vision is used to automatically obtain external environmental information obtained through technology, so it is necessary to carry out reasonable agricultural management. This process uses Kinect vision technology to identify crops. First, we need to segment the target from the background and obtain its position and distance information according to the target's characteristics. After processing this information, send it to the intelligent system. This information will generate corresponding instructions and perform corresponding operations. The Kinect depth sensing device can detect different levels of leaves, search for the leaves according to an iterative algorithm, mark different colors on the leaves of different levels, segment the leaves according to depth, and subdivide the rose leaves. Images can be output. The sensing device can quickly and accurately perform $3 \mathrm{D}$ measurement on the composition of rhizome leaves to obtain different curvatures of rhizome leaves. Kinect depth image real-time acquisition algorithm is applied in agricultural production as shown in Figure 4.

As can be seen from the data in Figure 4, the application of the real-time Kinect depth image acquisition algorithm in agricultural production will greatly increase the crop yield. The survey results show that the crop yield can be increased by about $12 \%$.

The Kinect image acquisition algorithm can obtain the distance information between the space object and the measuring device while acquiring the color image. The method of acquiring the depth information has been widely concerned, and it is also one of the researches in the field of agriculture on the acquisition of three-dimensional data of crops and other information fields-important research direction. Quantitative research on crop feature detection, from the two aspects of crop surface depth numerical measurement and horizontal projection area calculation accuracy analysis, demonstrated that Kinect's acquisition of images of plant trees, extraction of plant features, and detection of threedimensional data are achievable and effective and can meet the requirements of current agricultural applications. Using the basic Kinect depth image real-time acquisition algorithm, the accuracy error of the observed picture is about $0.24 \%$, and the relative error of the horizontal projection area is $0.76 \%$, which is very consistent with the actual observation. Its importance is selfevident. Kinect's observation data on reducing crop pest diseases is shown in Figure 5. 


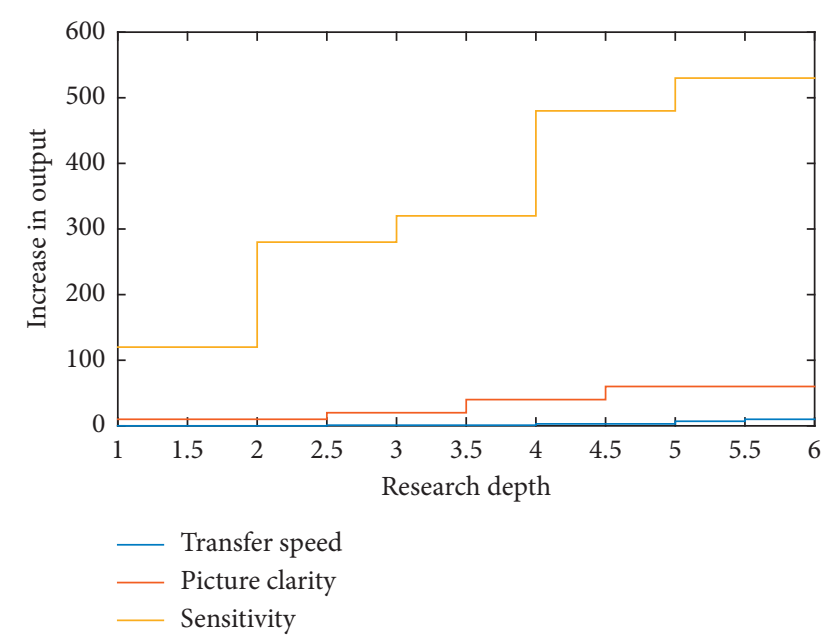

FIgURE 4: Kinect depth image real-time acquisition algorithm agricultural production application effect chart.

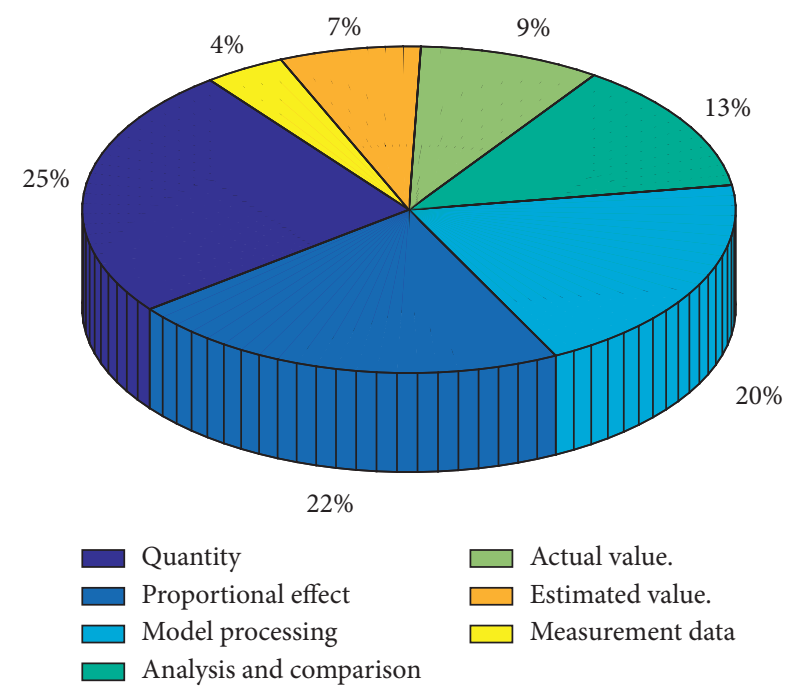

FIGURE 5: Kinect depth image real-time acquisition algorithm to reduce pest disease data analysis.

From the data in Figure 5, the real-time acquisition algorithm of Kinect depth images is very helpful for reducing crop pest diseases and can reduce the probability of crop pest diseases by $35 \%$.

\section{Conclusions}

In this study, the Kinect somatosensory equipment developed by Microsoft for indoor somatosensory games and human body parts and gesture recognition was used to try to expand the application to the outdoor real-time monitoring of crop growth in depth images and to carry out preliminary experiments. The test results show that the method has certain feasibility, innovation, and superiority. It has obtained good three-dimensional image data information and can provide valuable information for the three-dimensional reconstruction of crop growth models, analysis of growth status, and real-time monitoring of diseases and insect pests (data basis). With the development of modern agriculture more and more towards digitalization, refinement, and intelligence, fast, accurate and real-time acquisition of crop growth patterns and three-dimensional image information has gradually become an inevitable trend in future agricultural production management, and cheap, easy-to-use sensing equipment, like Kinect, with the continuous improvement of measurement accuracy will also reflect obvious advantages and gradually become an important tool in the field of agricultural image application research. This research mainly discusses the use of Kinect to obtain realtime outdoor crop growth depth images and 3D point cloud coordinate extraction. The next step will continue to use the obtained 3D image information for 3D construction, growth status, and application research of pests and diseases analysis.

\section{Data Availability}

No data were used to support this study.

\section{Conflicts of Interest}

The authors declare that they have no conflicts of interest.

\section{References}

[1] R. Samad, M. Z. Abu Bakar, D. Pebrianti, M. Mustafa, and N. R. H. Abdullah, "Elbow flexion and extension rehabilitation exercise system using marker-less kinect-based method," International Journal of Electrical and Computer Engineering (IJECE), vol. 7, no. 3, pp. 1602-1610, 2017.

[2] Q. Yang, H. Ye, and K. Huang, "Estimation of leaf area index of sugarcane using crop surface model based on UAV image," Transactions of the Chinese Society of Agricultural Engineering, vol. 33, no. 8, pp. 104-111, 2017.

[3] Z. Li, R. Pan, and J. Wang, "Real-time segmentation of yarn images based on an FCM algorithm and intensity gradient analysis," Fibres \& Textiles in Eastern Europe, vol. 24, no. 4, pp. 45-50, 2016.

[4] M. Liu, H. Li, and Zhu, "Design of real-time demosaickingzoomingimage acquisition system based on FPGA," Bandaoti Guangdian/Semiconductor Optoelectronics, vol. 36, no. 2, pp. 293-298, 2015.

[5] W. Yang, Z. Zhang, and Y. Zhang, "Real-time digital image stabilization based on regional field image gray projection," Journal of Systems Engineering and Electronics, vol. 27, no. 1, pp. 224-231, 2016.

[6] M. Ahmad, H. Nourzadeh, and B. Neal, "MO-FG-202-01: a fast yet sensitive EPID-based real-time treatment verification system," Medical Physics, vol. 43, no. 6, pp. 3711-3712, 2016.

[7] A. H. Basori, "Real time interactive presentation apparatus based on depth image recognition," International Journal of Electrical \& Computer Engineering, vol. 7, no. 3, pp. 13081315, 2017.

[8] M. Meng, F. Yang, and Q. She, "Human motion detection based on the depth image of Kinect," Chinese Journal of Scientific Instrument, vol. 36, no. 2, pp. 386-393, 2015.

[9] Z. Wu, Q. Chang, and M. Shang, "Two-dimensional parallel long code acquisition algorithm based on FFT," Journal of Terahertz Science \& Electronic Information Technology, vol. 13, no. 5, pp. 757-762, 2015. 
[10] Y. Jia, Z. Xu, Z. Su, S. Jin, and A. M. Rizwan, "Mosaic of crop remote sensing images from UAV based on improved SIFT algorithm," Transactions of the Chinese Society of Agricultural Engineering, vol. 33, no. 10, pp. 123-129, 2017.

[11] Y. Huang, X. Xu, and Yang, "ROI weld feature extraction algorithm of real-time tracking," Shanghai Jiaotong Daxue Xuebao/Journal of Shanghai Jiaotong University, vol. 50, no. 12, pp. 1877-1880, 2016.

[12] K. S. Raviya, D. V. Vyas, and A. M. Kothari, "Real time depth data refurbishment in frequency domain and 3D modeling map using microsoft kinect sensor," International Journal of Image Graphics \& Signal Processing, vol. 11, no. 11, pp. 49-58, 2016.

[13] S. Kumar and R. K. Jha, "An FPGA-based design for a realtime image denoising using approximated fractional integrator," Multidimensional Systems and Signal Processing, vol. 31, no. 4, pp. 1317-1339, 2020.

[14] Y. Dai, H. Zhang, D. Li et al., "Characteristic frequency acquisition algorithm of burst signal based on amplitude-time inflection point," Huanan Ligong Daxue Xuebao/Journal of South China University of Technology (Natural Science), vol. 46, no. 3, pp. 142-148, 2018.

[15] Y. Pan, T. Zhang, G. Zhang et al., "A novel acquisition algorithm based on two-dimensional delay estimation for BOC signals," Physical Communication, vol. 37, Article ID 100875, 2019.

[16] F. Zeng, X. Ouyang, H. Xu et al., "Improved long-code direct acquisition algorithm based on time-frequency fusion," Dianzi Yu Xinxi Xuebao/Journal of Electronics and Information Technology, vol. 41, no. 2, pp. 309-316, 2019.

[17] J. Dreier, M. Castello, G. Coceano et al., "Smart scanning for low-illumination and fast RESOLFT nanoscopy in vivo," Nature Communications, vol. 10, no. 1, pp. 556-577, 2019.

[18] X. Luo, R. Hu, and N. Hassan, "Denoising data acquisition algorithm for array pixelated CdZnTe nuclear detector," Open Physics, vol. 17, no. 1, pp. 144-152, 2019.

[19] C. L. Hwang and H. B. Abebe, "RGB-D face recognition using LBP with suitable feature dimension of depth image," IET Cyber-Physical Systems: Theory \& Applications, vol. 4, no. 3, pp. 189-197, 2019.

[20] Y. Su, W. Sun, J. Liu, G. Zhai, and P. Jing, "Photo-realistic image bit-depth enhancement via residual transposed convolutional neural network," Neurocomputing, vol. 347, pp. 200-211, 2019. 